\title{
Philosophiques
}

\section{Injustices raciales, responsabilité et déconstruction}

\section{Naïma Hamrouni}

Volume 40, numéro 2, automne 2013

URI : https://id.erudit.org/iderudit/1023707ar

DOI : https://doi.org/10.7202/1023707ar

Aller au sommaire du numéro

Éditeur(s)

Société de philosophie du Québec

ISSN

0316-2923 (imprimé)

1492-1391 (numérique)

Découvrir la revue

Citer ce document

Hamrouni, N. (2013). Injustices raciales, responsabilité et déconstruction. Philosophiques, 40(2), 465-471. https://doi.org/10.7202/1023707ar d'utilisation que vous pouvez consulter en ligne.

https://apropos.erudit.org/fr/usagers/politique-dutilisation/ 


\title{
Injustices raciales, responsabilité et déconstruction
}

\author{
NAÏMA HAMROUNI \\ Département de philosophie et des arts, Université du Québec à Trois-Rivières \\ naima.hamrouni@uqtr.ca
}

Sans distinction de race? de Magalie Bessone constitue l'une des preuves les plus éloquentes que la réflexion philosophique menée à un niveau conceptuel fondamental peut être mise au service d'un engagement politique fort, sans pour autant perdre en substance ni en sophistication. L'engagement politique ici, c'est celui qui vise l'élimination des injustices raciales. Il s'agit là d'un ouvrage important, salutaire dans le contexte de la France contemporaine où la question de la race semble être tombée sous la loi du silence, au moment même où le racisme gagne du terrain. Or, comme le souligne l'auteure, ne plus parler de la race, en rayer le mot dans la Constitution et en éliminer l'usage dans les enquêtes statistiques, comme l'ont réclamé de nombreux penseurs en France, ne feront pas magiquement disparaître les injustices raciales structurelles. Au terme d'une argumentation solidement menée dans un style analytique des plus clairs, et savamment étayée par des travaux issus de disciplines variées, l'auteure nous convainc dans ce livre que la lutte pour la justice raciale exige en tout premier lieu de reconnaître que la race, comme catégorie structurant l'organisation de nos sociétés, est toujours bien ancrée. Loin de réclamer la réhabilitation de ce concept sous son acception biologique courante, il s'agit plutôt — et tout l'enjeu est là de redéfinir la race à titre de catégorie socialement construite. Cela afin de pouvoir mieux penser les moyens politiques à mettre en œuvre pour combattre les injustices raciales.

Je partage l'essentiel des idées relatives au constructionnisme de la race défendues dans ce livre, d'autant plus que je souscris à une perspective critique similaire en ce qui a trait au genre dans mes propres recherches. Les points plus précis sur lesquels j'aimerais revenir succinctement dans le cadre de cette disputatio concernent davantage la philosophie politique de l'auteure. J'aborderai d'abord le problème de la condamnation morale dans un contexte d'injustices raciales structurelles. J'enchaînerai sur la critique très (trop ?) sévère adressée au multiculturalisme, dont les diverses tendances me semblent jusqu'à un certain point confondues. Je conclurai en posant à l'auteure une interrogation au sujet de la politique de la déconstruction dont je me revendique également, mais dans le cadre de recherches sur le genre.

\section{Culpabilité, responsabilité et injustice structurelle}

S'il n'y a pas encore de consensus autour de l'usage qui doit être fait du concept de race, tous s'entendent au moins pour considérer le racisme comme une faute morale méritant condamnation. Bessone s'oppose, au cinquième 
chapitre, à deux grandes conceptualisations du racisme, la première consistant à le réduire à sa dimension institutionnelle, la seconde, à une attitude psychologique individuelle. Elle veut défendre, contre ces deux tendances jugées réductrices, une approche dynamique et multidimensionnelle du racisme, qui intègre ces différentes dimensions et met l'accent sur la manière dont elles se renforcent et s'aggravent l'une l'autre. Si l'on ne peut qu'adhérer à ce projet visant une re-conceptualisation plus riche et fine du racisme, deux remarques peuvent cependant être faites. D'une part, malgré la volonté de défendre une conception multidimensionnelle du racisme, l'auteure en vient en fin de chapitre à mettre de l'avant une conception du «racisme comme manque de respect ", pleine de potentiel, mais qui risque justement d'avoir pour effet pervers l'évacuation du caractère "multidimensionnel» du concept. D'autre part, la critique que formule l'auteure à l'égard de la conception institutionnaliste du racisme me semble trop déterminante pour qu'elle puisse vraisemblablement récupérer cette dimension institutionnelle comme l'un des aspects de sa conception «multidimensionnelle». De plus, la raison invoquée pour disqualifier cette approche ne me paraît pas tout à fait convaincante. Je m'explique.

Parce qu'elle présente le racisme comme un trait strictement institutionnel, la conception institutionnaliste a conséquemment eu tendance à confondre racisme et discrimination systémique, nous dit l'auteure. L'un des effets pervers lié à cette confusion est qu'il n'est alors plus possible de procéder à la condamnation morale du racisme. En effet, le racisme institutionnel se reproduisant structurellement, sans que les agents soient nécessairement eux-mêmes racistes ou mal intentionnés, la question du coupable de l'injustice est laissée en suspens. Ainsi, pour ne pas que la "responsabilité morale des individus ou des collectivités» disparaisse tout à fait, Bessone suggère qu'il nous faut distinguer plus clairement racisme et discrimination systémique. Mais, loin d'offrir une réponse satisfaisante au problème de la condamnation morale, cette proposition risque d'avoir l'effet contraire: en laissant entendre que seuls les individus racistes sont moralement condamnables, l'auteure diminue considérablement la responsabilité individuelle et collective que nous avons dans le rétablissement de la justice. C'est ce que je voudrais souligner dans les lignes qui suivent.

Bessone me semble en fait souscrire à ce qu'Iris Marion Young a identifié dans son dernier livre comme étant le «modèle juridique de la responsabilité ", où la responsabilité se comprend en termes de culpabilité et où le rétablissement de la justice requiert absolument une condamnation morale ou juridique. Or, de plus en plus de penseurs ont soutenu que ce modèle n'était simplement plus adéquat pour appréhender la réalité contemporaine de nos sociétés démocratiques, libérales, post droits-civiques. Dans ces sociétés, l'injustice n'est pas que le fait de personnes particulièrement mal intentionnées qui contreviennent au droit, mais elle se reproduit plutôt de manière structurelle. Elle est le résultat d'une interaction complexe entre le 
fonctionnement des institutions, les processus de production et de consommation des biens et services, les comportements et les choix individuels. Le modèle juridique de la responsabilité, qui me semble mobilisé par l'auteure sans être nommé, ne permet pas de saisir ces injustices plus invisibles que jamais. Il perpétue la fausse division de la société entre victimes et bourreaux et laisse supposer que la «faute morale» pourra facilement être éradiquée une fois le coupable de racisme identifié et condamné. Comme l'écrit Young, ce modèle accentue indûment le pouvoir qu'auraient certains acteurs dominants et reporte démesurément la faute sur eux, tout en réduisant à néant la responsabilité des acteurs qui tiennent un rôle indirect dans la production de l'injustice (Young, 20II). Or la force de la conception institutionnelle du racisme (où bien entendu «l'institution» ne doit surtout pas être réduite à l'État) est qu'en échappant à la logique binaire et réductrice dominant/dominé, coupable/non coupable, elle permet d'appréhender les injustices raciales qui demeureraient invisibles sous le modèle juridique et de renouveler la conception de la responsabilité morale et politique pour la justice.

En ce sens, revenir à une conception réductrice de la responsabilité morale comme culpabilité, où seuls les individus racistes auraient une responsabilité morale ne me semble pas la voie à suivre dans le contexte contemporain. Au contraire, il importe d'élargir la notion de responsabilité de sorte que tous ceux qui sont impliqués de près ou de loin dans des structures productrices d'injustices puissent se voir imputer la responsabilité de s'engager à travailler en faveur de la réduction des injustices. Cette notion de «responsabilité relationnelle», plus intéressante et fructueuse, on la retrouve chez des philosophes comme Young. En somme, loin de faire disparaître la responsabilité morale et politique des agents, l'approche de la responsabilité relationnelle propose une conception extrêmement exigeante de la responsabilité morale et politique, mais surtout, une conception qui est à la mesure des injustices raciales structurelles qui aujourd'hui caractérisent notre monde social.

\section{Multiculturalisme et néoracisme}

Les discours essentialisants qui cherchaient depuis quelques siècles à inscrire la race dans la biologie humaine ont depuis été scientifiquement réfutés. Bessone perçoit toutefois dans le développement de certains courants de la recherche contemporaine un certain retour au discours racial naturalisant. Ce retour se constaterait à la fois dans le développement de tendances en sciences génétiques et en psychologie cognitiviste, où l'on a récemment postulé un "module» de pensée raciale qui serait universellement partagé. On est d'accord. Plus étonnamment toutefois, elle perçoit également cette tendance à l'essentialisation dans les théories du multiculturalisme, où un glissement vers un «naturalisme culturaliste» aurait littéralement été opéré. Je dis «plus étonnamment» parce que les penseurs du multiculturalisme ont 
un point commun important avec Bessone: ils ont adressé à la théorie républicaine française les mêmes critiques. Traiter tous les citoyens en tant qu'égaux en vertu de leur commune humanité, en niant leurs différences (de race, de classe, de genre, d'orientation sexuelle), revient de fait à privilégier systématiquement la perspective de la majorité, à abandonner les minorités à l'arbitraire des décisions majoritaires, et à masquer, sous le concept d'égalité et l'illusion de la neutralité, les injustices réelles dont elles sont victimes. Ils font donc le pari d'accorder une place à la différence culturelle et travaillent à l'élaboration et la justification de droits différenciés selon les groupes. Le problème, aux yeux de Bessone, est de deux ordres. D'abord, le multiculturalisme ferait un usage non critique de la différence, l'essentialiserait, et échouerait à en retracer la généalogie (la race est en réalité le produit d'une sombre histoire d'esclavage, de ségrégation, de discrimination, de colonisation dont aucune approche ne devrait faire fi). Ensuite, en mettant l'accent sur la culture (plus politiquement correct) plutôt que sur les races, il aurait contribué à redéfinir les catégories raciales à titre de groupes culturels minoritaires qu'il s'agirait alors de reconnaître, de célébrer, plutôt que de déconstruire. Ainsi, l'ère du multiculturalisme perpétuerait un "racisme» qui ne dit pas son nom, un «racisme sans races » qui consisterait à assurer la protection et la perpétuation des catégories raciales subtilement redéfinies comme groupes simplement culturels à reconnaître et consolider. La critique formulée mérite en effet toute notre attention et donne à réfléchir. Mais il serait requis de la nuancer. J'estime que si la critique de l'essentialisation de la différence peut s'adresser au courant différentialiste du multiculturalisme, elle s'applique beaucoup plus difficilement à la théorie libérale du droit des minorités, une théorie largement fondée sur l'argument de l'égalité morale des citoyens et la liberté de choix plutôt que sur celui de la valeur positive de la diversité culturelle. L'espace étant limité ici, je me contenterai de faire deux courtes remarques.

D'abord, contrairement à ce qui est suggéré, tous les multiculturalistes n'ont pas une conception totalement anhistorique des groupes sociétaux. L'approche libérale de Will Kymlicka, par exemple, comprend clairement les rapports de pouvoir et d'oppression à travers lesquels ces groupes (notamment les minorités nationales) ont pu se constituer, et qui persistent toujours. Et les droits différenciés selon les groupes qui sont mis de l'avant par cet auteur visent entre autres à assurer à leurs membres l'accès à un plus grand pouvoir et une protection externe contre l'arbitraire des décisions de la majorité. Il prévoit des droits spéciaux de représentation (dont l'objectif est de garantir la présence des minorités au sein des institutions de l'État), des droits à l'autonomie gouvernementale (visant la délégation de pouvoirs aux minorités nationales, par la mise en place du fédéralisme par exemple), et des droits polyethniques (qui garantissent un soutien financier et une protection juridique à certaines pratiques ou coutumes propres à des groupes religieux ou ethniques). Selon Bessone, les mesures de reconnaissance 
promues à l'intérieur des théories du multiculturalisme, comme la représentation dans les médias et dans les manuels scolaires, les exemptions à la loi ou encore la révision du récit de l'histoire nationale, auraient détourné notre attention des luttes, plus urgentes, contre la discrimination raciale, la persistance de la ségrégation, l'accès différencié au logement. Mais ces deux catégories de mesures politiques, en plus de pouvoir être adoptées et poursuivies simultanément, se renforcent aussi l'une l'autre. Pour prendre un exemple des plus banals, les droits d'exemptions à la loi en ce qui concerne le port de certains signes religieux dans la fonction publique et parapublique peuvent très bien s'inscrire dans une perspective visant à contrer la discrimination et la persistance de la ségrégation: l'abolition de la "race», et non sa célébration aveugle. Je terminerai en soulignant que la version libérale du multiculturalisme n'a jamais eu pour objectif de préserver ad vitam aeternam les traits particuliers ou le caractère d'un groupe culturel qui serait alors considéré comme une entité homogène, figée dans le temps. Il ne s'agit pas d'ériger des murailles infranchissables autour d'une communauté culturelle. La théorie de Kymlicka, encore, prévoit au contraire d'assurer la protection de la «structure" institutionnelle des cultures, le but étant de garantir, pour les citoyens, la capacité de "résister aux pouvoirs économiques ou politiques que la société majoritaire exerce sur [eux], assurer que les ressources et les institutions dont la minorité dépend ne sont pas tributaires des décisions de la majorité » (Kymlicka, 200I). Bref, supposer que la critique de l'essentialisation s'applique aussi bien au multiculturalisme libéral qu'à la politique de l'identité articulée autour de la valeur de la diversité, c'est, jusqu'à un certain point, faire fi des frontières idéologiques franches qui séparent ces deux tendances. Chez l'auteure, le rejet de la reconnaissance, au profit de la seule déconstruction, est radical. Ce qui me mène à demander: irait-elle jusqu'à soutenir qu'aucune injustice vécue en tant que membre d'un groupe (racial, social, de genre, d'orientation sexuelle, etc.) ne peut se décliner en termes de non-reconnaissance (parce que tous ces groupes seraient en réalité construits par les disparités de pouvoir et qu'abolir ces disparités abolirait du même coup ces différentes catégories sociales)?

On peut concéder à l'auteure que les questions raciales ont été négligées depuis les deux dernières décennies (elles l'ont toujours été d'ailleurs). La large place qu'occupait le multiculturalisme au-devant de la scène philosophique a peut-être eu pour effet de tenir ces enjeux dans l'ombre. On devrait même ajouter que les théories du multiculturalisme, de manière générale, ont passablement échoué à intégrer les analyses postcoloniales, et que le cas des minorités issues de l'immigration, qui sont souvent des minorités issues de la colonisation passée, n'a été traité que partiellement pour cette raison. Il paraît cependant exagéré de soutenir que toutes les théories du multiculturalisme ont entraîné une forme de néoracisme, ou pire, reposaient sur un racisme qui ne dit pas son nom. La conclusion qui doit être tirée des critiques formulées par l'auteure n'est pas, me semble-t-il, le rejet 
du multiculturalisme au profit des seules analyses critiques de la race. Si les groupes raciaux ne savaient être réduits ou assimilés aux groupes culturels, l'inverse est également vrai. Peut-être les deux groupes ont-ils été jusqu'à un certain point confondus, mais cette confusion n'est pas nécessaire. Ces critiques, à mon avis, font surtout réaliser à quel point il est nécessaire pour les théoriciens de la justice de pluraliser les outils conceptuels à partir desquels nous appréhendons les différentes formes d'injustices subies. Le multiculturalisme n'étant pas adéquatement outillé pour traiter de tous les enjeux relevant de la race, il nous faut réintroduire la catégorie raciale dans la réflexion philosophico-politique sur la justice. Je persiste à penser que le multiculturalisme libéral recouvre encore certaines dimensions des injustices subies par les minorités nationales et les minorités issues de l'immigration, des injustices de reconnaissance dont les analyses de la race ne sauraient rendre pleinement compte.

\section{Déconstruction et révolution}

Bessone se donne pour objectif dans ce livre de présenter le travail théorique et conceptuel préliminaire à la réflexion normative et politique ultérieure qui soutient la lutte pour l'atteinte d'une société a-raciale. Ce débroussaillage conceptuel doit ultimement nous «donner les moyens» de transformer notre société et de s'acheminer vers l'abolition de la race. La perplexité sur laquelle nous laisse toutefois le dernier chapitre vient justement du fait que l'approche déconstructionniste qui y est prônée (à l'encontre de l'approche multiculturaliste qui vise la valorisation identitaire des groupes racisés d'une part, et de l'approche républicaine-libérale qui nie la réalité sociale de la race au nom d'une fausse universalité d'autre part) ne semble en vérité déboucher vers aucune alternative politique. Quelles sont les implications politiques de l'approche théorique de la déconstruction? Que doit-on entendre par "politique de la déconstruction»? Ces questions que je pose à l'auteure en guise de conclusion font écho à celles que je me pose moi-même, qui me trouve dans un embarras similaire face aux implications politiques de l'approche déconstructionniste du genre. Je me contenterai de suggérer ici que, si la perspective théorique déconstructionniste critique se présente comme l'alternative la plus intéressante aux politiques simplement "réformistes» celles qui «trait[ent] des symptômes sans se préoccuper des causes» (comme l'écrit Bessone) - c'est aussi parce que l'abolition de la domination raciale visée par l'auteure implique une certaine forme de «révolution», bien que l'expression paraisse la rebuter et qu'elle s'en distancie aussitôt qu'elle doit l'évoquer. À partir du moment où l'on admet que l'égalité morale ainsi que l'exigence de non-domination et de parité de participation requièrent que tous ceux qui ont historiquement été exclus du contrat social, et dont l'inclusion récente n'a jamais été que purement nominale, puissent participer à égalité à la redéfinition des institutions de nos sociétés, à la détermination des valeurs qu'elles incarnent et de leurs règles de fonctionnement, du savoir 
qui porte sur elles et des mots pour en parler, nous devons reconnaître que le monde social, une fois la race (et le genre) abolis, ne sera plus jamais le même. Et que la portée de la justice réelle est beaucoup plus profonde qu'on n'ose l'admettre. Sans distinction de race? a toutes les ressources requises pour assumer son radicalisme. Il ne reste qu'à «ne plus avoir peur des mots », jusqu'au bout.

\section{Références}

Kymlicka, Will. La citoyenneté multiculturelle. Une théorie libérale des droits des minorités (trad. Patrick Savidan), Montréal, Boréal, 200r.

Mills, Charles W. et Carol Pateman. Contract and Domination, Cambridge, Polity Press, 2007.

Young, Iris Marion. Responsibility for Justice, New York, Oxford University Press, 2OII. 